\title{
Structure and Molecular Motion of Poly(ethylene oxide) Chains Tethered on Silica by Solid-State ${ }^{13} \mathrm{C}$ NMR Method
}

\author{
Katsuhiro Yamamoto, Atsushi Maruta, and Shigetaka Shimada \\ Nagoya Institute of Technology, Gokiso-cho, Showa-ku, Nagoya 466-8555, Japan
}

(Received February 13, 2001; Accepted April 28, 2001)

\begin{abstract}
Solid-state ${ }^{13} \mathrm{C}$ cross-polarization/magic-angle spinning/dipolar Decoupling (CP/MAS/DD) nuclear magnetic resonance (NMR) spectroscopy of poly(ethylene oxide) (PEO) tethered on silica was studied to characterize the conformation and dynamics of PEO chains. Temperature dependent NMR spectra could be interpreted in terms of the degree of interaction between PEO and silica molecules. The structures and molecular motion of the chains are strongly dependent on the grafting ratio (GR). Chemical shift of the methylene peak of the ${ }^{13} \mathrm{C}$ NMR spectrum increases with GR. $70.2 \mathrm{ppm}$ in low GR indicates "train" segments strongly interacted with the silica surface, whereas of $71.2 \mathrm{ppm}$ in high GR is originated from "tail" ("loop") segments separated from the surface. The fractional amount of "train" segment decreases remarkably with increase in GR. From the temperature dependence of the line width of the NMR spectra, correlation time, activation energy and transition temperature of the molecular motion were estimated. Molecular mobility of the "tail" segment was much higher and that of the "train" segment was lower than PEO chains in the amorphous region of the homopolymer bulk. "Tail" segments may thus protrude from the silica surface and have low segmental density, whereas "train" segments are trapped near the silica surface. It can be also considered that the fractional amount of "tail" segment increases abruptly with grafting ratio after the tethered chains of a coiled structure form one monolayer.

KEY WORDS Tethered Chain / Silica/Molecular Motion / Molecular Density / ${ }^{13} \mathrm{C}$ CrossPolarization/Magic-angle Spinning/Dipolar Decoupling (CP/MAS/DD) NMR /
\end{abstract}

Many investigations have been published on the adsorption of polymer on the silica surface. ${ }^{1-6}$ A few papers have been also presented about the adsorption behavior of polymer on the silica surface modified by different polymer chains. ${ }^{7,8}$ The development of hybrid materials comprised of organic and inorganic compounds can be expected. For example, when one polymer is grafted on the silica surface, another polymer miscible with the polymer can be mixed homogeneously. At present, it is very important to elucidate the structures and molecular motion of the tethered chains to discuss molecular compatibility of the two polymers in the blend system.

In our previous paper, ${ }^{9}$ the spin label method was used to study the structures and molecular motion of the end sites of the tethered poly(ethylene oxide) (PEO) chains on the silica. The conclusions were as follows: 1) The structures and molecular motion of the chain ends are strongly dependent on grafting ratio (GR). When GR $<\mathrm{GR}^{*}$ at which the overlap of the PEO chains starts, all PEO chains take a flat conformation ("train" conformation) and interact strongly with silica surface and molecular motion of the chains is suppressed. 2) When GR $>G^{*}$, the segments of the PEO chains released from the silica surface increase with GR, take "tail" conformation like a "polymer brush" and have high molecular mobility. 3) Chain ends of the tethered PEO chains in the structure like "polymer brush" have low segmental density and rotate rapidly in free space at high temperatures.

This paper clarifies the structures and molecular motion of the whole segments of the PEO chains tethered on the silica surface as a function of GR and confirms the above conclusions obtained by spin labeling.

Tajouri et al. ${ }^{10,11}$ studied the organization of a layer of PEO chains tethered on silica by measurement of ${ }^{1} \mathrm{H}$ spin-lattice relaxation and concluded that the chains adopted a flat conformation on the surface at low GR, whereas the chains adopted more extended conformation, perpendicular to the surface, at high GR. This paper characterizes the molecular mobility of the whole segments of the PEO chains and interactions between segments and silica by the ${ }^{13} \mathrm{C}$ NMR. The temperature dependency of chemical shift and line width of the PEO spectrum should give rise to dynamics and aggregation state of the segments in comparison with the dependency for PEO homopolymer bulk.

\section{EXPERIMENTAL}

\section{Materials}

Poly(ethylene oxide) PEO's having $M_{\mathrm{w}}=2.0 \times 10^{3}$ and $6.0 \times 10^{3}$ were purchased from Katayama Science Co. PEO's were purified by dissolving them in chloroform and precipitation in diethyl ether. The purified powder samples were dried under vacuum at room temperature for more than 1 day. The procedure was carried out twice. The silica gels ( $70-230$ mesh) were pur- 
chased from Nacalai Tesque Co. Specific surface area of the silica was $411.9 \mathrm{~m}^{2} \mathrm{~g}^{-1}$ as measured by BET (nitrogen adsorption) using NOVA21000 (Uasa Ionix Co.). The silica particles were purified by washing with acetone using a Soxlet apparatus and dried under vacuum at room temperature for more than 1 day.

\section{Preparation of PEO Chains Tethered on Silica Surface}

Grafting reaction was simply the direct esterification of a silica surface of silanol group by an hydroxy end of the PEO. ${ }^{12}$ The reaction was performed in polymer melt at $373 \mathrm{~K}$ to $453 \mathrm{~K}$ for $1 \mathrm{~h}$ in vacuum. The homopolymer was removed by the Soxlet extraction with acetone for 3 days. The grafted silica was dried under vacuum at room temperature for more than 1 day. PEO's having $M_{\mathrm{w}}=2.0 \times 10^{3}$ and $6.0 \times 10^{3}$ were grafted on the silica surface by this procedure. The grafted samples are called Si-g-PEO2K and Si-gPEO6K, respectively.

The grafting ratio, GR (weight ratio of PEO to silica) of each sample was determined by pyrolysis of weight loss between $373 \mathrm{~K}$ and $573 \mathrm{~K}$. Thermogravimetry (TG) measurements were carried out using a TG8101D Calorimeter Rigaku Denki Co. TG scans were run from $298 \mathrm{~K}$ to $773 \mathrm{~K}$ at heating rate of $5 \mathrm{~K}$ $\mathrm{min}^{-1}$. GR was calculated by the following equation, $\mathrm{GR}(\%)=($ PEO fraction $(\mathrm{g}) /$ Silica fraction $(\mathrm{g})) \times 100$.

\section{NMR Measurements}

High-resolution solid-state NMR experiments were carried out at $100.58 \mathrm{MHz}$ for ${ }^{13} \mathrm{C}$, using Varian UNITY plus 400 spectrometer. The instrument is equipped with a high-power amplifier decoupling and a Varian CP/MAS/DD (cross polarization/magic angle spinning/dipolar decoupling) probe. Spectra were referenced to the methyl carbon signal of tetramethylsilane (TMS) by taking the methyne carbon of solid admantane (29.5 ppm) as external reference standard. MAS frequency was set to $c a .4 .5 \mathrm{kHz} .4 .4 \mu \mathrm{s}, \pi / 2$ pulse was used for ${ }^{1} \mathrm{H}$ cross polarization pulse and proton decoupling strength was approximately $60 \mathrm{kHz}$. Contact time of CP signal enhancement was $0.5 \mathrm{~ms}$. Variation of the CP/MAS/DD spectra with temperature was observed. Temperatures in the sample, controlled by blowing cooled and warmed nitrogen gas, were calibrated using chemical shift of samarium acetate.

\section{RESULTS AND DISCUSSION}

\section{Structure of PEO Chains Tethered on Silica}

Figures 1 and 2 show the dependency of ${ }^{13} \mathrm{C}$ CP/MAS/DD NMR spectrum of PEO chains tethered on silica observed at $311 \mathrm{~K}$ on grafting ratio (GR).
Maximum peak shifts to down-field with increase in GR for both samples. The chemical shift $(\sigma)$ of 70.2 ppm observed for GR $=6 \%$ of PEO2K is remarkably lower than $71.2 \mathrm{ppm}$ and $72.0 \mathrm{ppm}$ assigned to PEO carbon in non-crystalline (amorphous) and crystalline phases, respectively, of PEO homopolymer bulk. ${ }^{13,14}$ The small value should be due to the strong interaction between the electric dipole of the PEO segments and silica molecules. Yoshino et al. observed the shift to high magnetic field of the ${ }^{13} \mathrm{C}$ NMR signal of methanol adsorbed on silica by increasing the concentration of the solvent and interpreted it in terms of interaction between methanol and silica molecules. ${ }^{15} \sigma$-Value is a decreasing function of interaction between ${ }^{13} \mathrm{C}$ and silica through oxygen atom. $\sigma$ is plotted with GR in Figure 3. The value, which reflects conformational structures of tethered chains, had a strong dependence on GR. $\sigma$ increased remarkably with GR and leveled off to $c a$. 71.2 ppm corresponding to the value in the non-crystalline phase of the PEO homopolymer bulk. This suggests

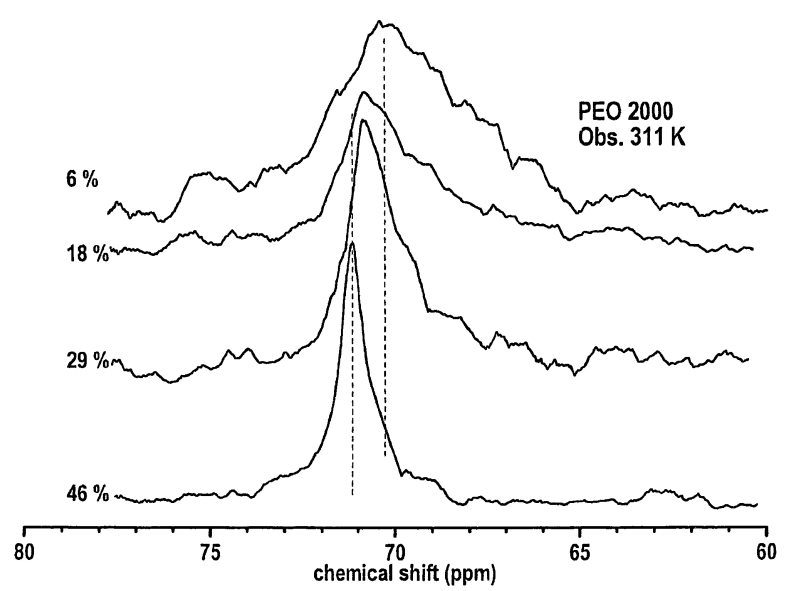

Figure 1. Variation of NMR spectrum of PEO tethered on silica surface with grafting ratio (GR) for Si-g-PEO2K. Measurements were carried out at $311 \mathrm{~K}$. Percent values in the figure are GR.

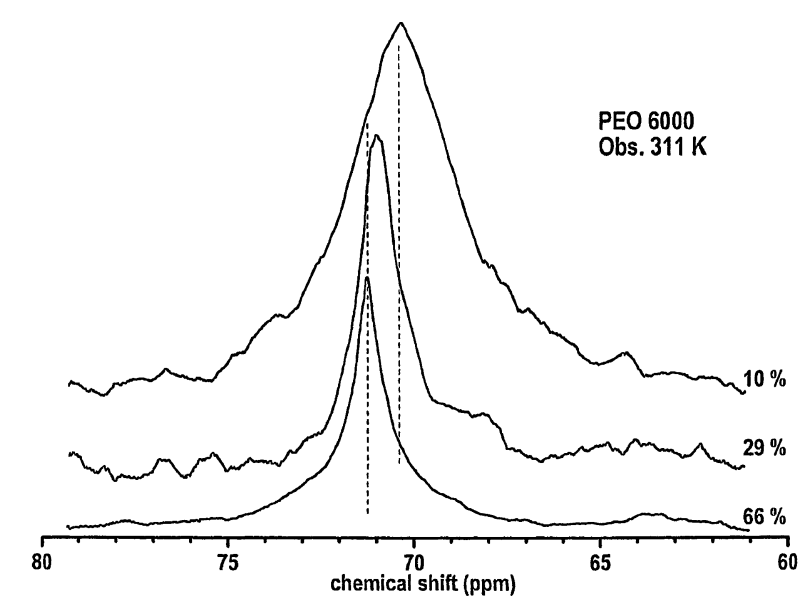

Figure 2. Variation of NMR spectrum of PEO tethered on silica surface with grafting ratio (GR) for Si- $g$-PEO6K. Measurements were carried out at $311 \mathrm{~K}$. Percent values in the figure are GR. 


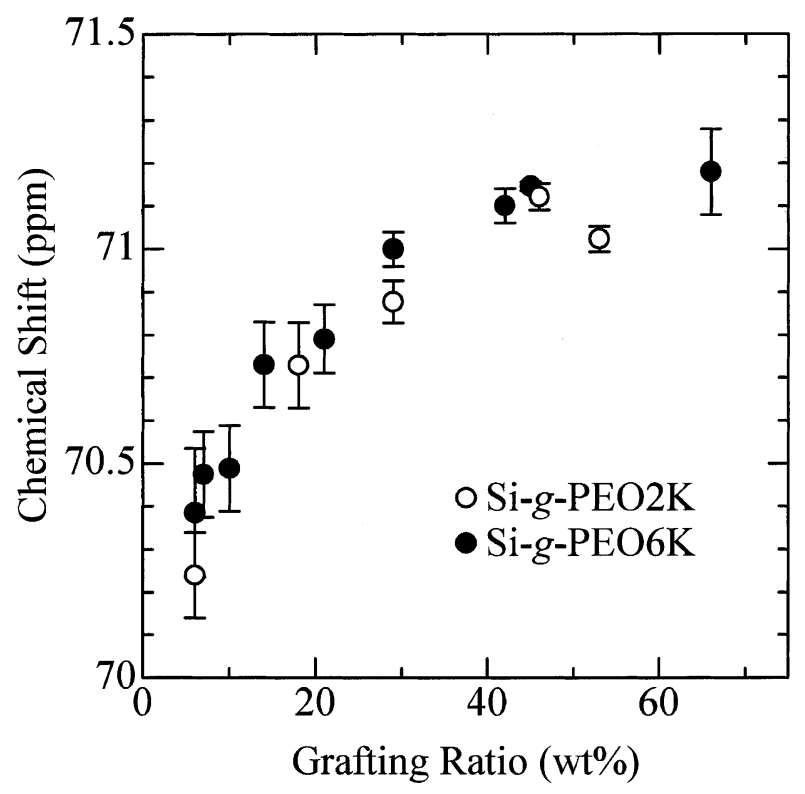

Figure 3. Variation of chemical shift $(\sigma)$ for SL-PEO tethered on the silica surface with grafting ratio. NMR measurements were carried out at $311 \mathrm{~K}$. Si- $g$-PEO2K (O), Si- $g$-PEO6K (

change of fractional amount of "train" to "tail" segment with GR. The segments of the tethered PEO chains having a low GR interact strongly with the silica surface and take a "train" conformation. With increase in GR, the tethered chains of a coiled structure cover the whole surface, form one monolayer and then, some segments protrude from the surface and take a "tail" ("loop") conformation with the contact of the tethered chains. All PEO segments depart from the silica surface and take a structure like a "polymer brush". $\sigma$ for the high GR is a function of interaction between PEO segments rather than PEO-silica interaction.

Theoretical analyse $\mathrm{e}^{16,17}$ of grafted polymer chains on the surface which take account of higher-order interaction predict the structure of a "polymer brush" because repulsion force steeply increases with graft density. Graft chains stretch away from the surface in higher graft density than $0.001-0.05$ chains $\mathrm{nm}^{-2}$. In our previous paper, ${ }^{9} \mathrm{GR}^{*}$ where PEO coils begin to overlap was approximately $11 \%$. The value of GR* corresponds to 0.09 chains $\mathrm{nm}^{-2}$ of graft density.

Yamamoto et al. ${ }^{18,19}$ prepared high-density polymer brushes comprised of poly(methyl methacrylate) chains densely end-grafted on a silicon substrate and clarified higher-order interactions among grafted chains from the long equilibrium thickness of the brush in toluene. Graft density on the silica substrate is larger than 0.4 chains $\mathrm{nm}^{-2}$. Graft density for $\mathrm{GR}=47 \%$ of Si-gPEO2K in the present paper corresponds to be 0.4 chains $\mathrm{nm}^{-2}$. PEO segments may thus take structures like the polymer brush.

Kawaguchi et al. ${ }^{20-22}$ concluded the preferential ad-

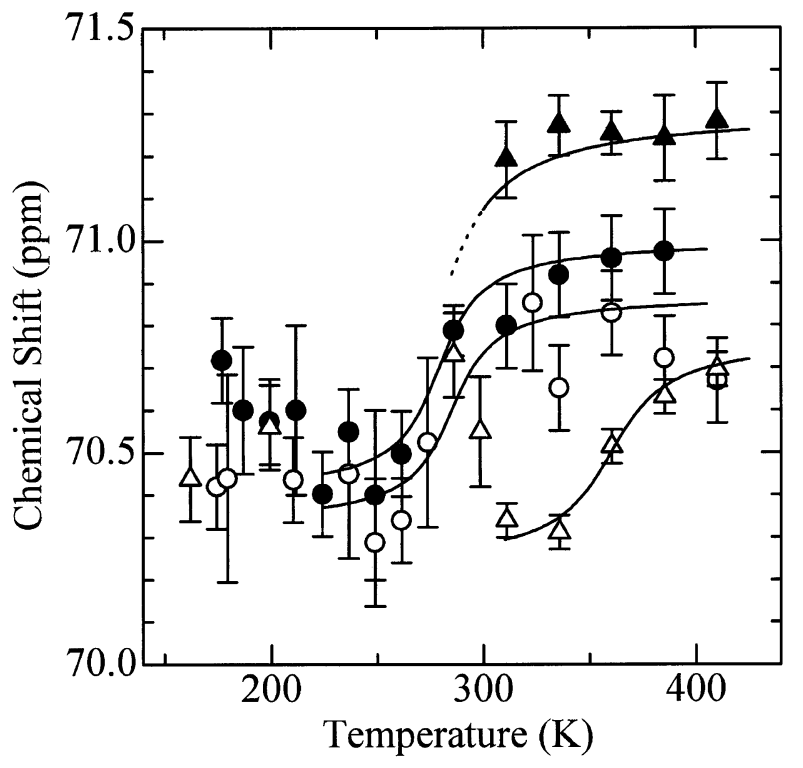

Figure 4. Temperature dependence of chemical shift $(\sigma)$ for SL-PEO tethered on the silica surface. Grafting ratio $(\mathrm{GR})=5 \%$ $(\bigcirc)$ and $47 \%(\bigcirc)$ of Si- $g$-PEO2K. GR $=10 \%(\triangle)$ and $66 \%(\mathbf{\Delta})$ of Si- $g$-PEO6K.

sorption of large molecules over small molecules. This indicates the large molecules have many sites of the polymer segment-silica interaction. However, molecular weight dependence of $\sigma$ reflects differences in average PEO segment-silica interaction for conformational structures of whole segments. The molecular weight dependence of the $\sigma$-value is ambiguous in Figure 3. Here, we conclude the fractional amount of "tail" ("loop") conformation increases with GR.

Is the dependence of $\sigma$-value on GR at $311 \mathrm{~K}$ in Figure 3 affected by molecular mobility of PEO segments? PEO segments of the "tail" ("loop") conformation at $311 \mathrm{~K}$ for high GR's should shrink, approach the silica surface and take a structure like "train" conformation at extremely low temperatures. We call the structure "quasi-train" conformation, the $\sigma$ of which is between those of the "train" and "tail" ("loop") conformation. Those of "train" conformation at $311 \mathrm{~K}$ should depart from the silica surface at higher temperatures than $311 \mathrm{~K}^{23}$

To confirm the conversion of "train" or "quasi-train" to "tail" ("loop") conformation, temperature dependence of $\sigma$ is estimated from the variation of NMR spectrum with temperature. $\sigma$ includes considerable error when the sample has an absolutely small quantity of PEO segment and the spectrum in a temperature range has a broad line width because of motional broadening as mentioned in the next section.

Figure 4 shows temperature dependency of $\sigma . \sigma$ for $\mathrm{GR}=5 \%$ of $\mathrm{Si}-\mathrm{g}-\mathrm{PEO} 2 \mathrm{~K}$ includes more experimental error because of the small quantity of PEO. Values at higher temperature tend to be larger than at lower tem- 
perature. The temperature dependency seems to be a transition phenomena. $\sigma$ for $\mathrm{GR}=10 \%$ of Si- $g$-PEO6K increases abruptly around $360 \mathrm{~K}$. This transition is a reflection of the conversion of "train" segments to "tail" ("loop") segments. The adsorbed segments begin to protrude from the silica surface and convert to "tail" ("loop") segments when the segments have thermal energy equal to the PS-silica interaction energy, which has a broad distribution. $\sigma$ for GR $=47 \%$ of Si- $g$-PEO2K increases abruptly around $275 \mathrm{~K}$. This transition is a reflection of conversion of "quasi-train" segments to "tail" ("loop") segments. The transition temperature of "train" ("quasi-train") to "tail" ("loop") conformation shifts to low temperature with increase in GR. This indicates weak interaction between the PEO segment and silica at low temperature for a sample of high GR. Extreme $\sigma$ at low temperature, where molecular motion is frozen, for high GR ( $47 \%$ of Si-g-PEO2K) is higher than at low GR (5\% of Si-g-PEO2K and $10 \%$ of Si- $g$ PEO6K). This indicates that PEO segments for high GR (Si-g-PEO2K and Si-g-PEO6K) take "quasi-train" conformation which has weak PS-silica interaction around the silica surface in comparison with the "train" conformation.

\section{Molecular Motion of PEO Chains Tethered on Silica}

To evaluate the molecular motion of tethered PEO segments related to PEO-silica interaction, NMR spectra were observed at various temperatures. Figure 5 shows the temperature dependence of NMR spectra of PEO chains tethered on silica having GR $=47 \%$ of Si$g$-PEO2K. As temperature increases, the line broadens at $224 \mathrm{~K}$, and narrows on further heating. ${ }^{13} \mathrm{C}$ line width of the $\mathrm{CH}_{2}$ carbon is plotted as a function of temperature in Figure 6. Hikichi et al. ${ }^{24,25}$ described how the onset of molecular motion associated with a glassrubber transition brought a temperature dependence to a ${ }^{13} \mathrm{C}$ line width in DD/MAS experiments and proposed

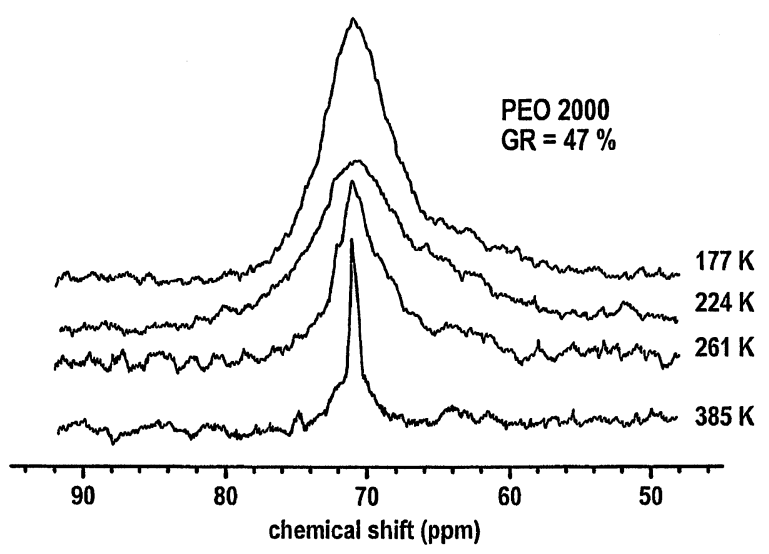

Figure 5. Temperature dependent NMR spectrum of PEO tethered on silica surface with grafting ratio of $47 \%$ of $\mathrm{Si}-g$-PEO2K. the following equation.

$\delta=\delta_{0}+\delta_{1}(2 / \pi)^{-1} \arctan \left(\alpha\left(T_{0}-T\right)\right)+\lambda M_{2}\left(\tau /\left(1+\omega_{1}^{2} \tau^{2}\right)\right)$

where the first term represents intrinsic line width arising from static line broadening. The second term describes motional averaging of the distribution of the isotropic chemical shift. $\alpha$ describes steepness of the narrowing. $T_{0}$ is the characteristic temperature for onset of molecular motion. The third term is motionalbroadening term and maximum broadening at $\omega_{1} \tau=1$ when $\tau$ is varied. $\lambda$ is a reduction factor and $M_{2}$ represents the power average of the second moment of the ${ }^{13} \mathrm{C}-{ }^{1} \mathrm{H}$ dipolar interaction. $\tau$ and $\omega_{1}$ are correlation time and ${ }^{1} \mathrm{H}$ decoupling frequency, respectively. Bimodal distribution of molecular mobility should be considered because motional modulation of ${ }^{13} \mathrm{C}-{ }^{1} \mathrm{H}$ dipolar interaction deteriorates the efficiency of crosspolarization. More mobile chains give a weaker NMR spectrum and may not contribute to line- broadening of the third term in the eq 1. Here, apparent mobility is discussed because the experimental spectra seem composed of only one component.

The average correlation time for segmental motion like a Micro Brownian motion should obey the WLF equation. For simplicity, we apply an Arrenius-type dependence of correlation time on temperature.

$$
\tau=\tau_{0} \exp \left(E_{\mathrm{a}} / R T\right)
$$

where $E_{\mathrm{a}}$ is activation energy and $\tau_{0}$ correlation time at infinite temperature. The empirical relationship suggested by Williams, Landel and Ferry (WLF) was interpreted in terms of the expansion of free volume in the matrices. Matsuoka et al. ${ }^{26,27}$ proposed a domain where number $(z)$ of conformers moves cooperatively and is to related the degree of cooperation with WLF equation. If equilibrium is achieved at low-temperature limit $T_{0}$, every conformer becomes meshed with the others and the number of conformers in one domain is nearly infinite. At high temperature-limit, they called $T^{*}$, each conformer relax independently of neighbors, and the number of conformer in each domain is 1 . Between the two extreme temperatures, the size of a domain is specified by $z$ in the domain which is a measure of cooperation of molecular motion. When the Arrenius-type dependence is applied, apparent activation energy, $E_{\mathrm{a}}$ has a physical meaning of $z \times \Delta \mu$ at an observation frequency or temperature. Here, $\Delta \mu$ means a rotational barrier of the $\mathrm{C}-\mathrm{C}$ bond and $z \times \Delta \mu$ should be closely related with the magnitude of the inter molecular interaction for the molecular motion. The dependence of the molecular mobility of the grafted chain on GR was elucidated by estimation of the apparent activation energy at the observation frequency. 
Table I. Best fitting parameters of $\mathrm{CH}_{2}$ line width of $\mathrm{PEO}$ tethered on the silica surface

\begin{tabular}{|c|c|c|c|c|c|c|}
\hline Samples & $\frac{E_{\mathrm{a}}}{\mathrm{kcal} \mathrm{mol}^{-1}}$ & $\begin{array}{c}\tau_{0} \\
\mathrm{sec}\end{array}$ & $\lambda$ & $\begin{array}{l}\delta_{0} \\
\mathrm{H}_{\mathrm{z}}\end{array}$ & $\begin{array}{c}\delta_{1} \\
\mathrm{H}_{\mathrm{z}}\end{array}$ & $\begin{array}{l}T_{0} \\
\mathrm{~K}\end{array}$ \\
\hline PEO (crystalline) & 9.3 & $1.0 \times 10^{-13}$ & 0.32 & 125 & 128 & 240 \\
\hline Si- $g$-PEO $(\mathrm{GR}=5 \%)$ & 9.3 & $1.3 \times 10^{-14}$ & 0.45 & 423 & 262 & 213 \\
\hline Si- $g$-PEO $(\mathrm{GR}=18 \%)$ & 6.5 & $2.4 \times 10^{-12}$ & 0.21 & 318 & 271 & 211 \\
\hline Si- $g-\mathrm{PEO}(\mathrm{GR}=47 \%)$ & 4.9 & $4.7 \times 10^{-11}$ & 0.21 & 308 & 257 & 192 \\
\hline
\end{tabular}

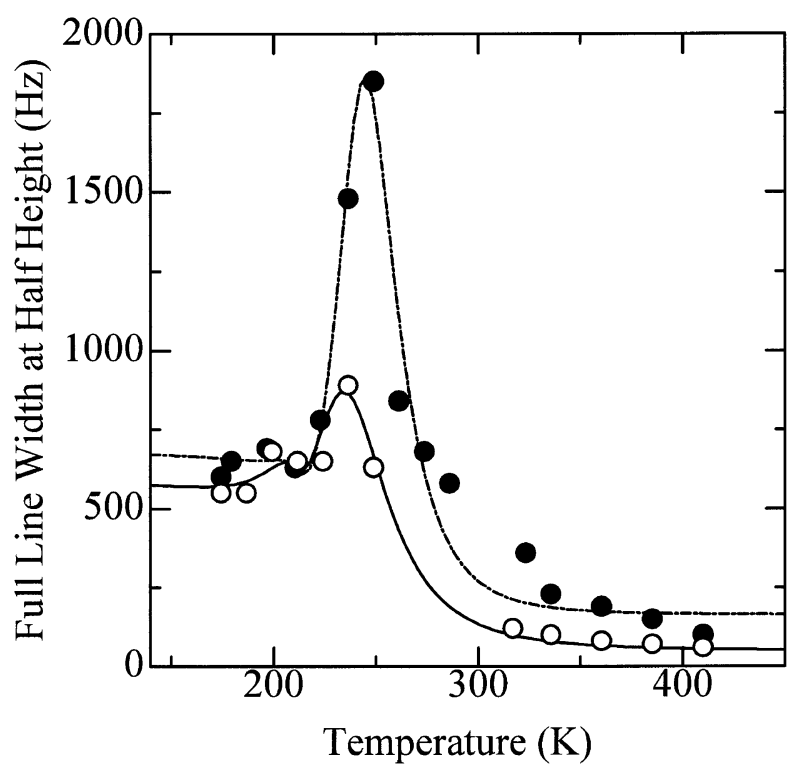

Figure 6. Variation of line width of NMR spectrum of PEO tethered on the silica surface with temperature for Grafting ratio $(\mathrm{GR})=5 \%(\bigcirc)$ and $47 \%(\bigcirc)$ of Si- $g$-PEO2K. Solid lines are obtained using eq 1 in text.

Observed line width was least-squares-fitted to eq 1 by taking $\delta_{0}, \delta_{1}, \alpha, T_{0}, \lambda, \tau_{0}$, and $E_{\mathrm{a}}$ as adjustable parameters. The solid lines through the data points in Figure 6 are "the best fit" ones. The agreement was excellent and the least-squares fitted parameters are listed in Table I.

Four parameters in the first and second terms in the eq 1 are evaluated ambiguously from the data points at lower temperature. The dependency of parameters of grafting ratio cannot be discussed. Maximum peaks were observed clearly and the third term in the eq 2 could be definitely evaluated. $E_{\mathrm{a}}$ and $\tau_{0}$ in the eq 2 can be determined with some accuracy by the "leastsquares-fitting". $\tau_{0}$ has no physical meaning when apparent activation energy changes with observation frequency. The large difference between $\tau_{0}$ for GR $=5 \%$ and $47 \%$ come from different values of $E_{\mathrm{a}}$. PEO segments may possibly move at a rate of $\omega_{1}=60 \mathrm{kHz}$ at the temperature where line width has a maximum in Figure 6. PEO segments in Si-g-PEO2K having higher $\mathrm{GR}=47 \%$ are much more mobile than at low $\mathrm{GR}=$ $5 \%$ in Figure 6 . The activation energy of molecular motion decreases with increase in GR as shown in Table I. Figure 7 shows temperature dependence of line width for the sample having GR $=18 \%$ in comparison

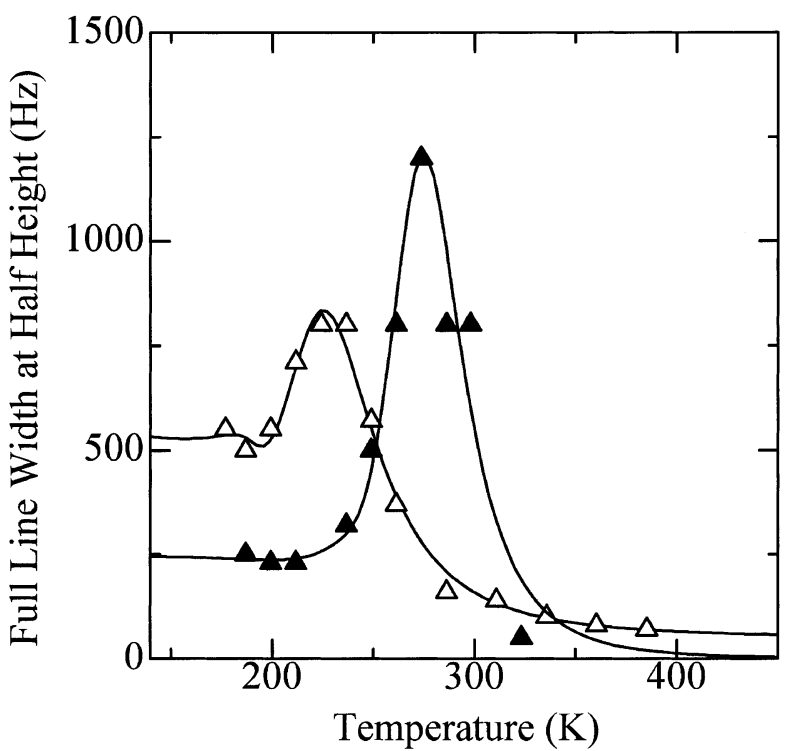

Figure 7. Variations of line width of NMR spectrum of PEO in the homopolymer, Bulk $(\boldsymbol{\Delta})$ and tethered on the silica surface with temperature for Grafting ratio $(\mathrm{GR})=18 \%(\triangle)$ of Si- $g$-PEO2K. Solid lines are obtained using eq 1.

with PEO homopolymer bulk, where the NMR spectrum of ${ }^{13} \mathrm{C}$ in the crystalline phase, not in the amorphous phase, was observed under the experimental conditions. The lower temperature at the maximum peak for Si-g-PEO2K (18\%) than that for the homopolymer bulk indicates that tethered chains are much more mobile than PEO chains in the crystalline phase. Arrenius type dependency on temperature is depicted in Figure 8 using determined $E_{\mathrm{a}}$ and $\tau_{0}$ shown in Table I. The temperature (O), where Micro Brownian type molecular motion in the PEO homopolymer bulk ${ }^{28}$ occurs at $\omega_{1}=60 \mathrm{kHz}$ is plotted in the figure. The characteristic temperature was estimated from a relaxation map of PEO, by Wada. ${ }^{28}$ The relaxation map in Figure 8 indicates that molecular mobility of the tethered PEO segment in Si- $g$-PEO2K having GR $=5 \%$ is lower than in the amorphous phase of the PEO homopolymer bulk. Mobility of PEO segments in the silica having GR $=18 \%$ and $47 \%$ is higher than in PEO bulk. Tethered chains having a low GR are adsorbed strongly on the silica surface and take flat conformation. With increase in GR, chains contact each other, protrude from the surface and take "tail" ("loop") conformation and finally all chains stretch perpendicularly on the silica 


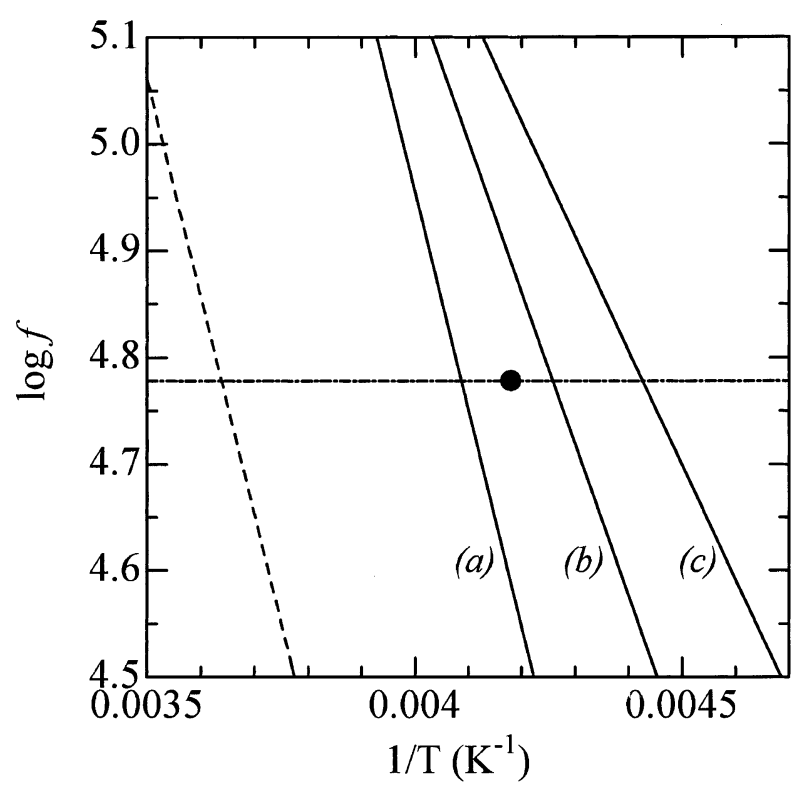

Figure 8. A relaxation map of PEO tethered on the silica surface. Dependence of relaxation frequency $(f)$ on inverse temperature $(1 / T)$ Grafting ratio (GR) $=5 \%$ (a), $18 \%$ (b) and $47 \%$ (c) of Si- $g$-PEO2K. Dashed line is temperature dependence of PEO in the crystalline region calculated from NMR data and $(\mathbf{)})$ is relaxation frequency in the amorphous region of PEO bulk, obtained from map.

surface like a "polymer brush". Changes of the structure and molecular motion with GR obtained from the NMR data are consistent with ESR data in our previous paper. ${ }^{9}$

\section{CONCLUSION}

The structures and molecular motion of PEO chains tethered on silica surface are strongly dependent on the grafting ratio (GR).

1) When GR $<G^{*}$ at which overlap of the PEO chains starts, all PEO chains take flat conformation ("train" conformation) and interact strongly with the silica surface and molecular motion of the chain is suppressed.

2) When GR $>G^{*}$, the segments of the PEO chains released from the silica surface increase with GR, take "tail" conformation like a "polymer brush" and have high molecular mobility.

3) Transition of "train" ("quasi-train") conformation to "tail" ("loop") conformation is related to interactions between PEO segments and the silica sur- face.

\section{REFERENCES}

1. A. Takahashi and M. Kawaguchi, Adv. Polym. Sci., 46, 1 (1982).

2. J. V. Alster and D. M. Ovenall, Polym. Commum., 32, 549 (1991).

3. A. K. Chakraborty and P. M. Adriani, Macromolecules, 25, 2470 (1992).

4. J. E. Gambogi and F. D. Bulum, Macromolecules, 25, 4526 (1992).

5. A. A. A. Ei-Hakim, A. M. Ramadan, and A. S. Badran, Polymer, 33, 4880 (1992).

6. H. D. Bijsterbosch, M. A. C. Stuart, and G. J. Fleer, Macromolecules, 31, 981 (1998).

7. S. Shimada, K. Horiguchi, and K. Yamamoto, Colloid Polym. Sci., 276, 412 (1998).

8. S. J. Sofia, V. Premnath, and E. N. Merrill, Macromolecules, 31, 5059 (1998).

9. S. Shimada, A. Maruta, and K. Yamamoto, Polymer J., 32, 1038 (2000).

10. T. Tajouri and H. Bouchriha, Polymer, 37, 3185 (1996).

11. S. Azrizi, T. Tajouri, and H. Bouchriha, Polymer, 41, 5921 (2000).

12. H. Hommel, A. P. Legrand, H. B. Ouada, H. Bouchrihat, H. Baland, and E. Papirer, Polymer, 33, 181 (1992).

13. X. Zhang, K. Takegoshi, and K. Hikichi, Macromolecules, 26, 2198 (1993).

14. S. Schantz, Macromolecules, 30, 419 (1997).

15. Private communication.

16. S. T. Milner, Science, 251, 905 (1991).

17. P-Y. Lay, Macromolecules, 24, 4981 (1991).

18. S. Yamamoto, E. Muhammad, Y. Tsujii, and T. Fukuda, Macromolecules, 33, 5608 (2000).

19. S. Yamamoto, E. Muhammad, Y. Tsujii, M. Matsumoto, and T. Fukuda, Macromolecules, 33, 5602 (2000).

20. M. Kawaguchi and T. Arai, Macromolecules, 24, 881 (1991).

21. M. Kawaguchi, S. Anada, K. Nishikawa, and N. Kurata, Macromolecules, 25, 1588 (1992).

22. S. Anada and M. Kawaguchi, Macromolecules, 25, 6824 (1992).

23. S. Shimada, Y. Hane, and T. Watanabe, Polymer, 38, 2315 (1997).

24. K. Takegoshi and K. Hikichi, J. Chem. Phys., 94, 3200 (1991).

25. T. Miyoshi, K. Takegoshi, and K. Hikichi, Polymer, 38, 2315 (1997).

26. S. Matsuoka and X. Quan, Macromolecules, 24, 2770 (1991).

27. S. Matsuoka, "Relaxation Phenomena in Polymers", Hanser Publishers, Munich, Germany, 1992, chapt. 2.

28. Y. Wada, J. Phys. Soc. Jpn., 16, 1226 (1961). 